\title{
Measurement of neutrino interaction ratio between water and hydrocarbon using a new detector with a 3D grid structure
}

\author{
K. Kin , Y. Azuma, T. Inoue, Y. Seiya, K. Yamamoto \\ on behalf of the J-PARC T59 Collaboration \\ Osaka City University \\ 3-3-138 Sugimoto, Sumiyoshi-ku, Osaka-Shi, Osaka, Japan \\ E-mail: kenichidocupcl.hep.osaka-cu.ac.jp
}

\begin{abstract}
In the T2K experiment, there are systematic errors caused by uncertainties of neutrino-nucleus interaction due to the target difference between a far-detector (water) and near-detector (hydrocarbon). To reduce these errors, we plan to measure the neutrino interaction ratio between water and hydrocarbon with an accuracy better than 3\% at J-PARC. A new detector consists of $3 \mathrm{D}$ grid-like scintillators to achieve $4 \pi$ solid angle acceptance. To read out signals, new electronics was developed with the France LLR group. Construction work was finished, and the detector was installed into the T2K near-detector hall. After the installation work, we confirmed that the detector was working very well by using cosmic-rays. Also, we observed well-reconstructed cosmic-muons. We started the neutrino beam measurement in October 2017. We successfully observed neutrino events. The detector is working very stably. The data taking are continued until May 2018, and $8.0 \times 10^{20}$ POT are expected to be accumulated. We expect to observe about 1000 signal events. In this paper, the details of a new detector and the status of the neutrino beam measurement will be reported.
\end{abstract}


Measurement of neutrino interaction ratio between water and hydrocarbon using a new detector with a $3 D$ grid structure

\section{Introduction}

\subsection{Motivation}

The T2K is a long-baseline neutrino oscillation experiment that uses intense beams produced at J-PARC. In this experiment, neutrino oscillation parameters are determined by measuring the neutrino flux before oscillation at the near-detector, ND280, and after oscillation at the fardetector, Super-Kamiokande (SK). The neutrino target of ND280 is mainly hydrocarbon (partially water), while that of SK is water. Due to this difference of the targets, uncertainties of neutrinonucleus interaction do not cancel, which causes systematic errors of the oscillation analysis at T2K. Moreover, SK has a $4 \pi$ solid angle acceptance, while ND280 can mainly measure forward scattering events. The difference in acceptance also contributes to systematic errors. In order to suppress such systematic errors $\left(4.2 \%\right.$ for $v_{e}$ appearance / $2.9 \%$ for $v_{\mu}$ disappearance $\left.{ }^{[1]}\right)$, we construct a new detector to precisely measure the neutrino cross-section ratio between water and hydrocarbon with the (anti-)neutrino beam at J-PARC ${ }^{[2]}$. This measurement is important for the $\mathrm{T} 2 \mathrm{~K}$ to confirm the $\mathrm{CP}$ violation in the lepton sector with more than $3 \sigma$ significance.

\subsection{Detectors}

From the upstream side of the neutrino beam, hydrocarbon target detector, water target detector, and muon-range detector are placed (Fig. 1). The water target detector is newly constructed for our measurement, and others are exsisting detectors in the T2K. The new detector has a 3D grid-like structure of plastic scintillators (Fig. 2). Thanks to this structure, the detector has $4 \pi$ slid angle acceptance and high-ratio water volume (80\%). Using these detectors, we can derive the neutrino cross section from the equation:

$$
\sigma=\frac{N_{\text {signal }}-N_{B G}}{\varepsilon T \varphi}
$$

where $\sigma$ is the cross section, $N_{\text {signal }}$ is the number of signal events, $N_{B G}$ is the number of background events, $\varepsilon$ is the efficiency, $T$ is the number of nuceus in the target material, and $\varphi$ is the neutrino flux. Our goal is to measure the neutrino cross-section ratio between water and hydrocarbon with an accuracy better than $3 \%$.

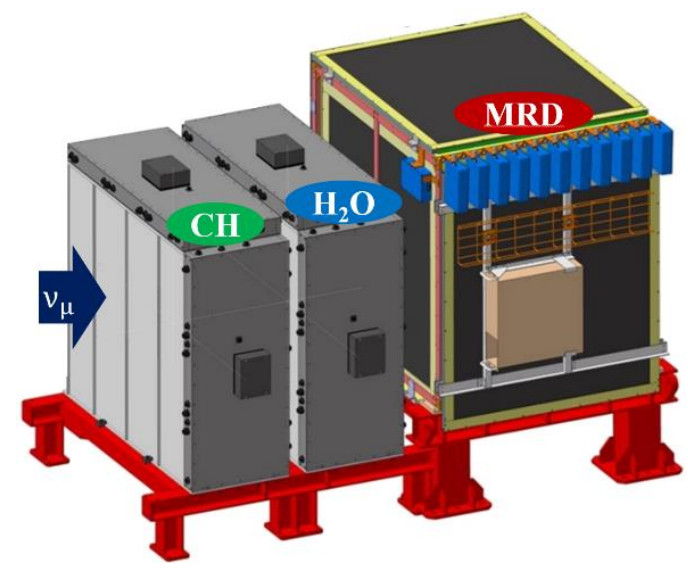

Figure 1: Illustration of detectors.

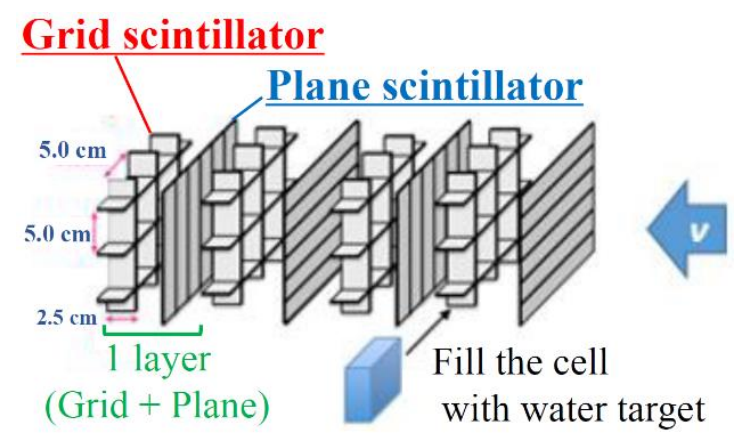

Figure 2: 3D grid structure. 
Measurement of neutrino interaction ratio between water and hydrocarbon using a new detector with a $3 D$ grid structure

\subsection{Expected number of events}

The number of events in the new detector is estimated by the Monte Calro simulation and summarized in Table 1. Charged-current (CC) neutrino interaction is the signal event in SK. High statistics and low background are expected.

Table 1: Expected number of events in $1.0 \times 10^{21}$ POT (POT: Proton On Target).

\begin{tabular}{|c|c|c|c|c|}
\hline & CC interaction & NC interaction & BG from outside & Total \\
\hline \# of events & 31466 & 1608 & 1832 & 34906 \\
\hline Fraction [\%] & 90.1 & 4.7 & 5.2 & 100 \\
\hline
\end{tabular}

\section{Water detector construction}

At first, scintillators and WLS fibers were glued with the optical cement. These scintillators were painted with reflector and black spray to suppress the crosstalk effect. Then we aligned these scintillators in a 3D grid-like structure (Fig. 3). Four scintillator layers were assembled into one sub-module, and 4 sub-modules were also assembled into one module (Fig. 4). The module was installed into a water tank. The detector was installed into the T2K near-detector hall as shown in Fig. 5

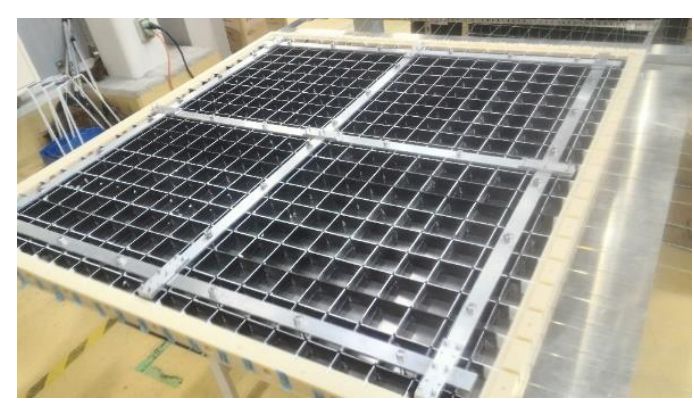

Figure 3: Grid scintillator layer.

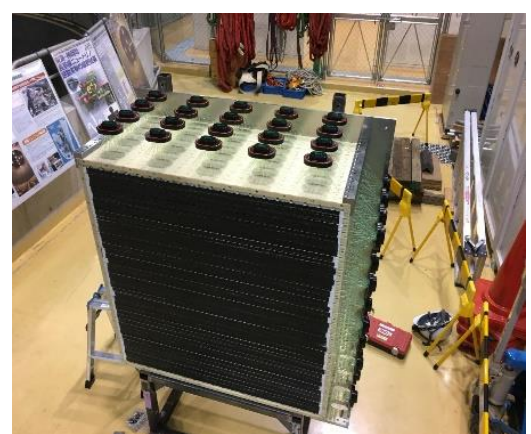

Figure 4: Grid scintillator layer.

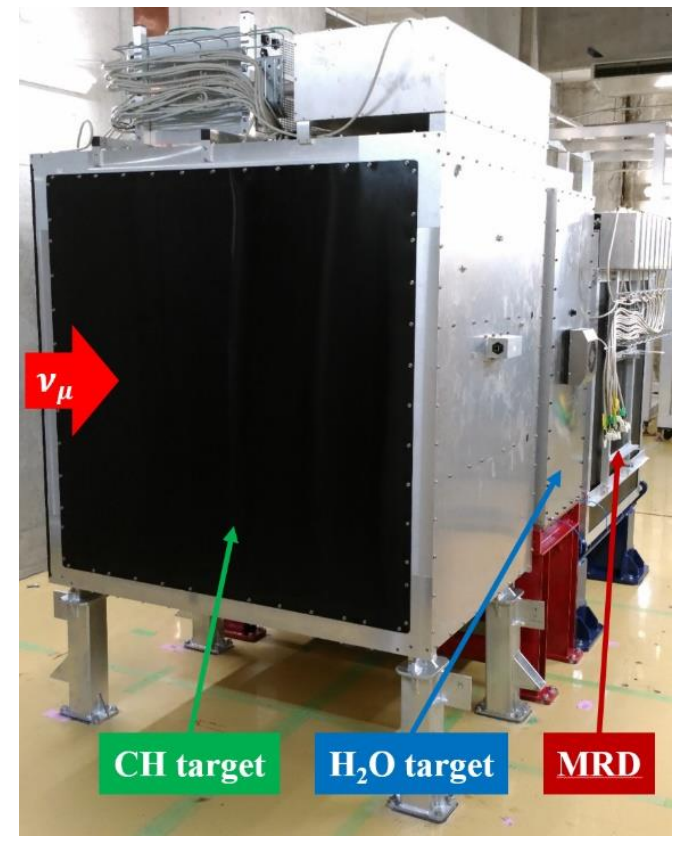

Figure 5: Installed detectors into the T2K near-detector hall. 
Measurement of neutrino interaction ratio between water and hydrocarbon using a new detector with a $3 D$ grid structure

\section{Signal read-out system for the new detector}

We develop new electronics for the new detector with the France LLR group. A schematic view of electronics is shown in Fig. 6, and the details of each component are summarized in Table 2. In this system Multi-Pixel Photon Counters (MPPCs) are used to read out signals of charged particles. The MPPCs and ASU boards with new ASIC, SPIROC2D ${ }^{[2]}$, are attached on the detector as shown in Fig. 7.

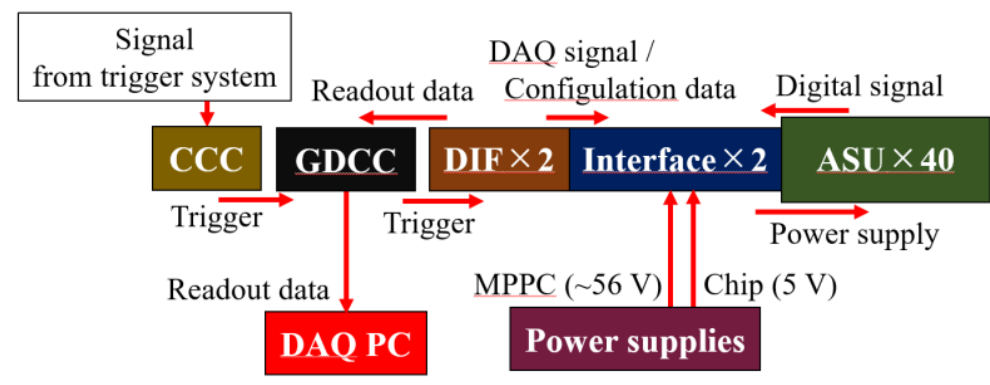

Figure 6: Schematic view of new electronics.

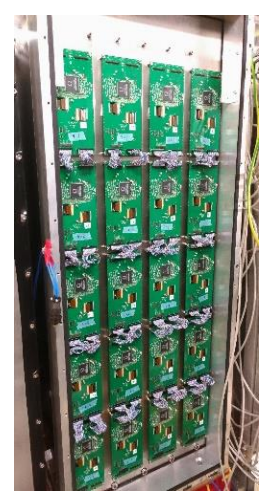

Figure 7: ASU boards.

Table 2: Details of electronics components.

\begin{tabular}{|c|c|}
\hline Components & Description \\
\hline ASU & Readout signal from MPPC w/ SPIROC2D chip \\
\hline Interface & Transfer DAQ signal and MPPC bias voltage \\
\hline DIF & Send DAQ signals and chip configuration \\
\hline GDCC & Transfer signals between DAQ PC and DIF \\
\hline CCC & Provide clock signals \\
\hline
\end{tabular}

\section{Commissioning by using cosmic-rays}

After the detector installation, commissioning work was performed by using cosmic-rays.

\subsection{MPPC performance}

For all the MPPCs (1280 channels), we checked the ADC distribution. We observed not only pedestal peaks but also 1 p.e. peaks as shown in Fig. 8. There were no dead channels after the installation work. We also checked the distributions of gains after tuning them (Fig. 9). The variation of gains was small enough, $<10 \%$.

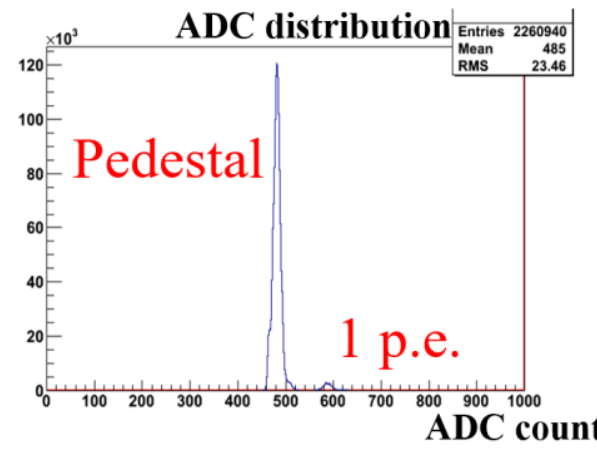

Figure 8: ADC distribution of MPPC.

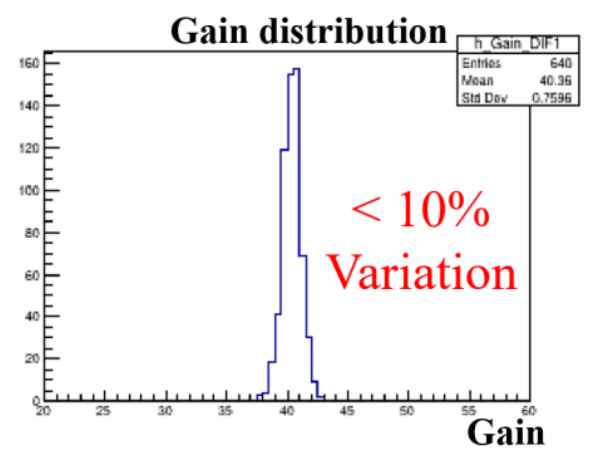

Figure 9: Gain distribution after the gain tuning. 
Measurement of neutrino interaction ratio between water and hydrocarbon using a new detector with a $3 D$ grid structure

\subsection{Light yield of scintillators}

Light yield of all the 1280 channels were checked by cosmic-rays (Fig. 10). The mean light yield was about 25 p.e., and it was enough to detect charged particles produced by neutrino interactions. Only 3 channels had light yield less than 15 p.e., and they had low light yield before the detector installation.

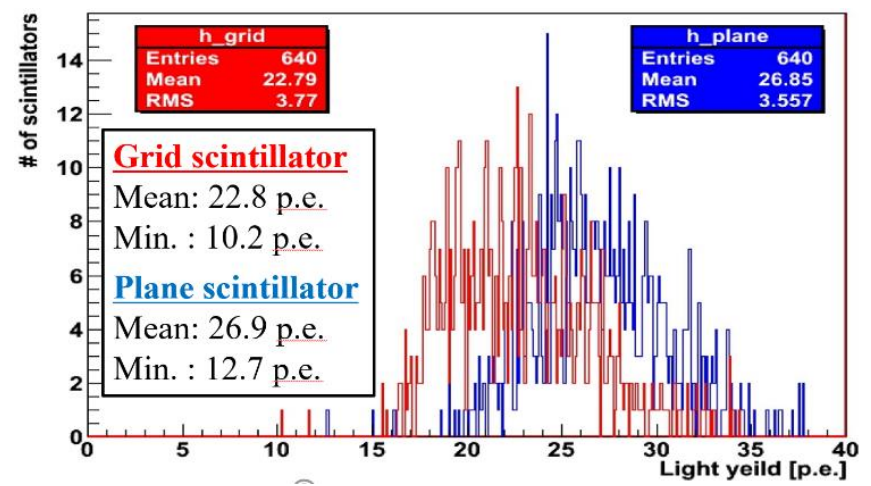

Figure 10: Light yield of 1280 channels by cosmic-rays.

\subsection{Event display}

As shown in Fig. 11, we successfully observed well-reconstructed muons in the event display. From this result, we also checked that the channel mapping had no problem.

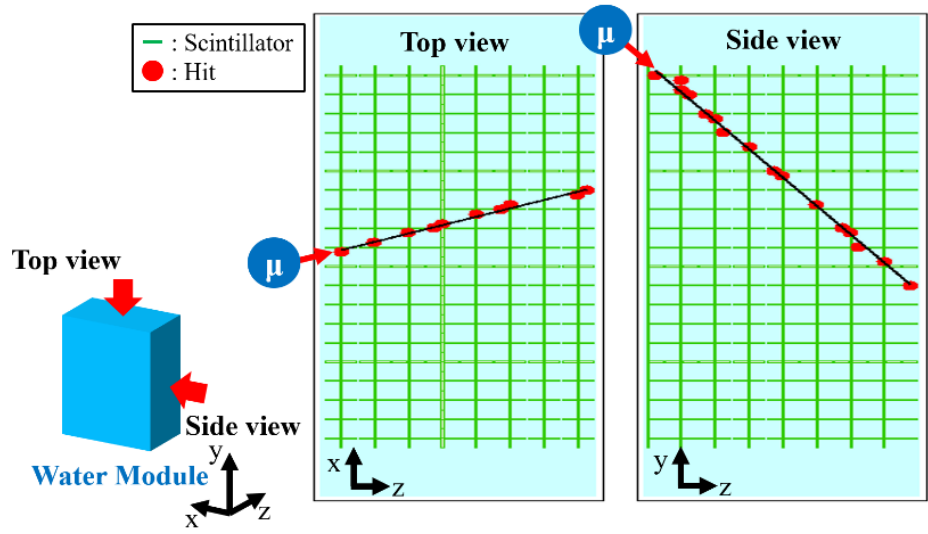

Figure 11: Event display of cosmic-muon.

\section{Neutrino beam measurement from October 2017}

\subsection{Expected statistics}

We started the anti-neutrino beam measurement at J-PARC in October 2017. We expect $8.0 \times 10^{20}$ POT to be accumulated until May 2018. Using these data, about 1000 signal CC events are expected to be observed after the event selection. So far, about $3.0 \times 10^{20}$ POT have been accumulated. 
Measurement of neutrino interaction ratio between water and hydrocarbon using a new detector with a $3 D$ grid structure

\subsection{Detector performance}

We successfully observed the first neutrino event (Fig. 12) on 17th October 2017. We are continuing to take data without serious problems. Gains for all the MPPCs are stable and no new $\mathrm{bad} / \mathrm{dead}$ channels are found. Light yields for all the scintillators are also stable. There is no water leak from the water tank so far. The detector is working very stably.
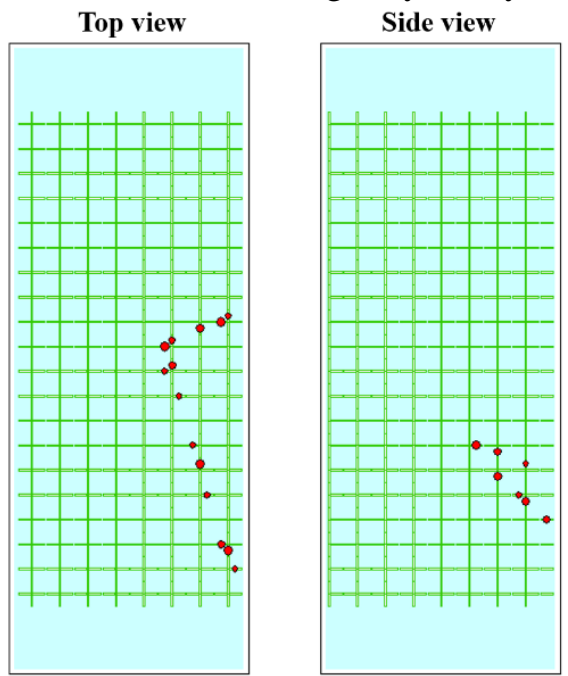

Figure 12: Event display for the first neutrino event.

\section{Summary}

Measurement of neutrino cross-section ratio between water and hydrocarbon is ongoing at J-PARC to reduce the systematic errors in the T2K neutrino oscillation analysis. We have constructed a new water detector which had a 3D grid-like structure, and installed it into the detector hall. We checked the MPPC performance, light yield of scintillators, and event display of reconstructed muons by cosmic-rays. We started the anti-neutrino beam measurement at JPARC in October 2017. We successfully observed the neutrino events and the detector is working very stably. By the end of May 2018, 8.0 $\times 10^{20}$ POT will be collected and about 1000 signal events are expected to be observed.

\section{References}

[1] K. Abe et al. (T2K Collaboration), Phys. Rev. D 96, 092006 (2017).

[2] K. Kin, Proceedings of 27th International Conference on Neutrino Physics and Astrophysics (NEUTRINO 2016), J. Phys.: Conf. Ser. 888012125 (2016)

[3] Omega, SPIROC2D User guide (2009) 